\title{
Promoting Global Health through Improved Blood Collections and Transfusion Practices: A Pilot Project between Rush University and University of Global Health Equity in Rwanda
}

\author{
Gillard LA ${ }^{1 *}$ and Royeen $\mathrm{CL}^{2}$ \\ ${ }^{1}$ Department of Medical Laboratory Science, Rush University, USA \\ ${ }^{2}$ Dean, College of Health Sciences, Rush University, USA
}

*Corresponding author: Laurie Gillard, Rush University, 600 South Paulina Street, AAC 1018, Chicago, Illinois, 60612 USA, Tel: 312-942-2402; Email: Laurie_Gillard@rush.edu

\section{Review Article}

Volume 5 Issue 2

Received Date: October 06, 2021

Published Date: November 25, 2021

DOI: $10.23880 /$ phoa-16000190

\section{Abstract}

This paper will first review what constitutes Sub-Saharan Africa (SSA) and the current blood banking practices therein. The recommendations by the World Health Organization (WHO) for implementing voluntary blood donation as well as the many challenges of testing for transfusion transmitted infectious disease will be described. To support a quality system in blood transfusion services, the introduction of national blood standards through the establishment of the National Blood Transfusion Services (NBTS) and the creation of the African Society for Blood Transfusion will also be delineated. Finally, to continue this critically important work, collaboration between Rush University (Chicago) and the University of Global Health Equity (Rwanda) has been initiated and is described. The goal is to move towards bringing advanced blood banking knowledge to the continent of Africa though the Specialist in Blood Bank Educational Program using distances education. This small but innovative project adds to improved global health.

Keywords: Transfusion Safety; Transfusion Transmitted Infection; Malaria; Pathogen Reduction Technology; Blood Safety; Volunteer Donation

Abbreviations: SSA: Sub-Saharan African; WHO: World Health Organization; EML: Model List of Essential Medicines; NBTS: National Blood Transfusion Services; AfSBT: Africa Society for Blood Transfusion; WB: Whole Blood; TTI: Transfusion Transmitted Infection; OH: Obstetric Hemorrhage; IV: Intravenous; HBV: Hepatitis B; HCV: Hepatitis C; HIV: Human Immunodeficiency Virus; RDT: Rapid Diagnostic Test; EIA: Enzyme Immunoassay; NAT: Nucleic Acid Testing; TTM: Transfusion Transmitted Malaria; PRT: Pathogen Reduction Technology; EU: European Union; US: United States; PEPFAR: The President's Emergency Plan for AIDS Relief; UGHE: University of Global Health Equity.

\section{Introduction}

In middle and low income Sub-Saharan African (SSA) countries, blood collection and transfusion practices do not have the support and resources that are available in many of the high income countries worldwide. Although progress has been made to address resulting health inequities, the collection of blood and the consequential testing and its transfusion do not always follow the World Health Organization's recommendations for best practice. This article will provide an overview of the challenges that exist within SSA including donor collection (replacement verses volunteer), the risk of transfusion transmitted infection and ways to mitigate it, and an introduction to the National Blood Transfusion Services (NBTS) and the Africa Society for Blood Transfusion (AfSBT) to support blood bank quality management though national accreditation. The final section will summarize collaboration between Rush University (Chicago) and the University of Global Health Equity (Rwanda) to address the inequity of blood availability and transfusion in Africa through an innovative distance 
education program.

\section{Sub-Saharan Africa}

Africa is a huge continent and consists of 54 countries, with 46 of them sub-Saharan. According to the United Nations, the sub-Sahara consists of all African countries and territories that are fully or partially located south of the Sahara. In 2015 The World Health Organization (WHO) created a Model List of Essential Medicines (EML) and the Guidelines on management of blood and blood components as essential medicines [1]. The EML emphasizes the need to establish regulatory oversight of production of blood to assure the quality, safety and efficacy for their use in transfusion. Operationalization of transfusions is particularly relevant to sub-Saharan Africa (SSA) since whole blood remains the predominate transfused product. Thus, for developing countries with limited resources, moving from the use of whole blood (WB) to specific blood components requires a huge commitment mostly from the government and other organizations (i.e., WHO, European Union, AABB) to help create an environment where patients would have access to a range of specialized and safe blood products. In SSA the availability and safety of blood transfusion using whole blood is clearly a huge concern due to the prevalence of HIV/ AIDS, HCV, and malaria as well as emerging infections, such as Ebola.

\section{The Current Status of Blood Availability}

Many countries in SSA have established a national blood transfusion service (NBTS) using the WHO recommendations and guidelines. Approximately 35 of the SSA countries have reported having a national blood policy. Although there are gains towards the establishment of NBTSs, having a policy doesn't guarantee the countries have published standards of blood collection, processing, testing and distribution. National guidelines and applicable blood safety policies and governmental support and funding are not adequate [2]. Blood shortages remain frequent in this region since more than 40 countries have failed to attain the WHO's donation goal of 10 units/1000 population. One study demonstrated a 19\% increase in WB donation in 12 African countries from 2011-2014, however, this increase has not been sustained [3].

Most transfusions in the sub-Sahara are whole blood and red cells and are used for disease related anemias, malaria, hookworm, and HIV/AIDS related bleeding disorders, obstetrics (anemia of pregnancy and obstetric hemorrhage), trauma, and burns. Interestingly, the strategy to prevent, minimize, or avoid transfusion is through the understanding of the mechanisms of anemia and coagulopathy, which is a large part of patient blood management using evidencebased practices. Providing access to best practices would benefit the physician and the patient within sub-Saharan Africa. In one study $25 \%$ of obstetrical hemorrhage patients did not have blood available. Another study reported $52 \%$ mortality in severely anemic children who were not transfused within 8 hours of diagnosis [4]. WHO targeted $1-2 \%$ of each country's population to donate blood. However, current figures are closer to 10 donations per 1000 people or less than $0.01 \%$. The resulting shortages of blood create dire situations at hospitals resulting in delayed treatment for patients. The lack of availability or the provision of unsafe blood severely impacts patient morbidity and mortality.

\section{Volunteer vs. Replacement Blood Donors}

Many countries, (i.e., Namibia, Zambia, and Botswana) have made great progress towards the goal of $100 \%$ volunteer donors but there still are not enough volunteer blood donors to support the need. In the US all blood donations come from volunteer donors [5]. This is not the case, however, in the sub-Sahara. Replacement donation is still the most common type of donation. Individuals providing replacement donation blood are often paid by family members and friends to donate for the patient. Because the motivation to donate is a financial one, there is a much higher risk of transfusion transmitted infection (TTI). Women remain underrepresented in the donor pool due to the cultural perception that men are healthier than women. Further, pregnancy and iron deficiency anemia also contribute to the lower number of female donors [6].

\section{The Urgent Need for Safe Transfusion: Sickle Cell Disease and Peripartum Blood Loss}

Nearly $80 \%$ of the 300,000 children born with sickle cell diseaseworldwide occur in Sub-Saharan Africa. In high income countries like the United States and the United Kingdom over $95 \%$ of these children will reach adulthood because of access to early diagnosis and treatment. In some African countries, 50 to $90 \%$ of those children will not survive to their $5^{\text {th }}$ birthday [7]. Obstetric hemorrhage $(\mathrm{OH})$ and access to peripartum blood transfusion remains a huge challenge. The rates of peripartum transfusion in South Africa exceed those in high-income countries despite comparable rates of $\mathrm{OH}$. The major risk factors are prenatal anemia and access to prenatal care, with HIV and infection related moderately low platelet counts as other complications. Women who are anemic are less able to tolerate even small to moderate hemorrhage during delivery. Iron deficiency anemia is the most common cause of prenatal anemia in South Africa [8]. These outcomes suggest better interventions to diagnose and correct prenatal anemia would help avoid transfusions. 
Blood transfusion is critical to obstetric resuscitation. Earlier diagnosis and better treatment of prenatal anemia, including intravenous (IV) iron therapy, could have a substantial impact on the necessity of transfusion for these women.

\section{Transfusion Transmitted Infections (TTI)}

Blood safety remains a huge issue in sub-Saharan Africa. Patient safety is compromised by the use of unevaluated diagnostic reagents and the lack of infrastructure and logistics of supplies (temperature, controlled storage, etc.). A study evaluated blood borne virus testing and transfusion safety in Africa and it revealed the poor overall sensitivity of blood screening especially for Hepatitis B (HBV), Hepatitis C (HCV), and human immunodeficiency virus (HIV) [9]. Biomedical testing of blood donation for HIV, HBV and HCV and syphilis has expanded, however, in a 2016, only 6 of 46 surveyed African countries reported 100\% screening coverage for the major TTIs. Most of the testing was performed using rapid diagnostic tests (RDT). The RDTs are used because they are lower cost and uncomplicated to use even though they have lower sensitivities and specificities than enzyme immunoassay (EIA) and nucleic acid testing (NAT) [9]. Donor screening for HBV and HCV have increased over the past 20 years however, $23 \%$ of the global burden of HBV is in SSA [10].

\section{Transfusion Transmitted Malaria}

Transfusion transmitted malaria (TTM) is a neglected subject in SSA where malaria is endemic and not well studied [11]. In endemic regions, a mosquito could also have bitten a person who received a blood transfusion that contains malaria parasites. To determine whether the source of malaria parasites is from the unit of blood transfused or an earlier mosquito bite requires genotyping which is not routinely performed. Further, in endemic countries the presence of parasitemia does not mean the donor has malarial disease, since people living in malaria endemic countries develop partial immunity after repeated exposure to infectious mosquito bites. Such individuals can carry parasitemia without developing the clinical disease. Asymptomatic parasitemia may or may not progress to clinical disease. Clinical disease is observed in patients with chills, fever, headache and vomiting in the presence of Plasmodium parasitemia. Asymptomatic blood donors pose a risk and happen to be the most typical blood donors in sub-Saharan Africa [11]. All persons living in malaria endemic areas are susceptible to malaria infections, but only certain populations are especially susceptible such as children under 5-years-old and pregnant women. Infants under 6 months old appear to be protected from malaria due to passive antibodies from the mother. The prevalence of parasitemia in blood donors varies across Africa and it dependent upon how endemic it is in the country and region and the transmission season.

Ideal screening tests for plasmodium in donors or donated blood do not presently exist. Microscopy is not sensitive because "healthy" donors have low parasitemia. Nucleic acid testing (NAT) would be ideal but it's too expensive for SSA. Even if the blood center could screen for malaria, the blood banks and transfusion services can't afford to discard $50 \%$ of the already low blood supply. Rapid detection tests are used but have low sensitivity with low parasitemia. Antibody testing cannot be used because nearly all adults have been exposed to plasmodium and will have a positive result.

The WHO recommends that donor selection criteria should be developed in endemic countries to identify and collect blood from donors at the lowest risk, both during the malaria session and during the rest of the year. A few countries defer donors in Africa. In Zimbabwe where malaria is not endemic, the approach is to screen donors by asking if they have visited a malaria area within the previous 21 days [12]. If they have and did not take prophylaxis, the donor is deferred for 6 weeks. If they visited a malarial region and contracted malaria, they are deferred for 6 months after they receive a full course of treatment and have been found to be clinically symptom free.

\section{Bacterial Contamination of Blood}

Bacterial contamination of blood products (especially platelets) is largely neglected in Africa. There isn't always adherence to sterile technique during blood collection and processing in hot, humid conditions encourages bacterial growth. Additionally, inadequate refrigeration increases the risk of bacterial contamination of red cells and whole blood. Diversion pouches and blood culture, which are commonly used in high income countries, are not routinely used in resource-limited countries. Studies have shown bacterial contamination rates range between $2 \%$ and $17 \%$ in SSA compared to an estimated 1 in 3000 units in the United States [13].

\section{In Vitro Treatment of Donor Blood to Reduce Transmission of Infectious Agents}

In vitro treatment has been done in two ways: either using anti-malarial drugs or the use of Pathogen Reduction Technology (PRT). There are two anti-malarial drugs, quinine and sulfadoxine-pyrimethanime that have been experimentally used and found to be effective against parasites in blood bags. There is also concern of bacterial 
contamination of the blood by entering the bag and the risk of increasing the resistance to the antimalarial drug being used. The most promising development is using PRT. There have been clinical trials using Marisol (one form of PRT) in whole blood, which not only inactivates viruses but it also inactivates the plasmodium [14].

In SSA, with few exceptions, donor testing is serological testing for HIV, HCV, and HBV. Implementing molecular testing methods is limited by cost, technical expertise, limited infrastructure, and regulatory oversight. Pathogen reduction is a term used to describe a variety of methods (solvent detergent treatment or photochemical activation) and can be applied to blood following collection. It's a way to diminish the infectious potential of multiple pathogens simultaneously. Improved blood transfusion safety could result from the implementation of PRT but the main challenges are it would have to be cost effective (even in developed countries it's expensive). Further, pathogen reduction technology requires trained operators and it requires regulatory oversight. PRT was developed to compliment current testing in North America but in Africa it could ultimately be an alternative to testing. The benefits of PRT are amplified in SSA compared to high resource countries where NAT testing and irradiation are readily available. This technology better suits the countries where the TTI testing is less developed because it has the potential to improve blood safety by reducing or eliminating infectious organisms, including bacteria, viruses, and parasites, from blood components intended for transfusion.

\section{World Health Organization (WHO), National Blood Transfusion Services (NBTS) and the Creation of the Africa Society for Blood Transfusion (AfSBT)}

Over the last 15 years, many SSA countries have been technically and financially supported by the WHO, the European Union (EU), the United States (US), and other external foundations. In the US, The President's Emergency Plan for AIDS Relief (PEPFAR) which began in 2003, was created to assist in the development of an infrastructure including dedicated facilities for blood transfusion services [15]. The PEPFAR program included the development of national standards (The National Blood Transfusion Services) and strengthened the implementation of a quality system in blood transfusion services, including information management.

In many countries in SSA, the government does not support blood collection organizations. Because of this, a strong infrastructure for collection, personnel training, infectious disease testing, and ultimately, building a base of volunteer donors is lacking. In 2017, the WHO published a Strategic framework for blood safety and availability 2016 -2025 [16]. This guidance provides a framework for interventions and is intended to help African countries (as well as other WHO member countries) to develop an organized, sufficiently funded blood transfusion system with clearly defined regulatory oversight. The goal is to collect blood and blood components from voluntary, nonremunerated donors from low risk populations and perform quality assured testing of all donated blood, including screening for TTIs and associated compatibility testing. In addition, the WHO guidance supports the implementation of an effective quality management system based on a national quality policy and includes a framework for action for blood safety and availability. The Africa Society for Blood Transfusion (AfSBT) was created with the guidance of the WHO. The AfSBT developed assessment criteria for a regulatory system using a tiered approach to accreditation to enable blood transfusion services that do not meet all the requirement standards, to receive partial accreditation while working towards full accreditation [17]. The mission of the AfSBT is to advocate for the highest ethical and professional standards and skills in blood transfusion across the African continent, enabling safe, universally accessible and sustainable national blood programs in participating countries [18] .

\section{Advancingthe PromiseofSaferTransfusions: The Partnership: Rush University and the University of Global Health Equity}

The partnership between Rush University (Chicago), and the University of Global Health Equity (UGHE) (Rwanda), was established in 2019, to enhance the current medical and healthcare education being offered at UGHE. With the support of a generous donor, the Specialist in Blood Banking (SBB) program at Rush University was selected as a pilot program based on the need for advanced blood banking and transfusion practice education in Africa. The Rush SBB program is online and asynchronous and given the different time zones, works well with individuals attending the program remotely in Rwanda. The course work is presented in weekly modules over a period of 12 months. The curriculum of the SBB program covers best practices in all areas of blood banking and transfusion medicine, from donor eligibility and collections through infectious disease testing, blood distribution, transfusion, blood utilization, and patient blood management. This educational alliance between Rush University and UGHE is in position to support the WHO Strategic framework for blood safety and availability 20162025 [16]. The WHO advocates Member States to establish effective and sustainable blood transfusion services and to create a national quality management system throughout 
the blood supply spectrum, from the donor vein to the patient vein. The UGHE graduates of the SBB program will be prepared to take leadership roles in these critical patient/ donor activities which are listed in the WHO Strategic framework for blood safety and availability 2016-2025: Annex to Resolution EM/RC63/R.5 [16].

Once the UGHE graduate completes the SBB program, they are considered subject matter experts in blood banking and transfusion medicine and will contribute to the development of advanced blood banking and transfusion practice curriculum as well as teach the curriculum to medical students at UGHE. The overarching goal of the Rush University and UGHE partnership is to create African blood bank experts who will contribute to improved global health, support health equity, and become the future leaders in transfusion excellence for Africa.

In conclusion, the need for advanced blood banking initiatives, including education, cannot be over emphasized. Blood and blood products are considered drugs because of their therapeutic use for numerous medical conditions. Progress in transfusion medicine stresses transfusion safety, the pursuit of public safety and the availability of high quality blood and blood products. The WHO and the AfSBT are addressing the need for regulation of the processes involved in blood product collection and manufacturing. Rush University and the University of Global Health Equity are poised to supply the educational expertise and future subject matter experts to support safer transfusion practices and a more equitable health system in Africa.

\section{Acknowledgements}

This paper was supported, in part, through the A. Watson Armour III Presidental Professor endowment granted to the second author.

\section{Conflicts of Interest}

None

\section{References}

1. WHO (2021) WHO Model Lists of Essential Medicines. The World Health Organization.

2. Weimer A, Tagny CT, Tapko JB, Gouws C, Tobian AAR, et al. (2019) Blood transfusion safety in sub-Saharan Africa: a literature review of changes and challenges in the 21st century. Transfusion 59(1): 412-427.

3. Chevalier MS, Kuehnert M, Basavaraju SV, Bjork A, Pitmanet JP (2016) Progress toward strengthening national blood transfusion services-14 countries, 2011-
2014. MMWR Morb Mortal Wkly Rep 65(5): 115-119.

4. Kiguli S, Maitland K, George EC, Olupot-Olupot P, Opoka OR, et al. (2015) Anemia and blood transfusion in African children present to hospital with sever febrile illness. BMC Med 13: 21.

5. Barro LL, Drew VJ, Poda GG, Tagny CT, El-Ekiaby M, et al. (2018) Blood transfusion in sub-Saharan Africa: understanding the missing gap and responding to present and future challenges. Vox Sang 113(8): 726736.

6. Swanevelder R, Ravi R, Dhuly C, Olmsted M, Brambilla D, et al. (2019) Using a motivator and deterrent questionnaire to predict actual donation return behavior among firsttime African-origin blood donors. Transfusion 59(9): 2885-2892.

7. Thompson AA (2017) Making a case for more sickle cell initiatives in Africa. Blood 129(2): 136-137.

8. Bloch EM, Ingram C, Hull J, Fawcus S, Anthony J, et al. (2018) Risk factors for peripartum blood transfusion in South Africa: a case control study. Transfusion 58(9): 2149-2156.

9. Laperche S (2013) Multinational assessment of bloodborne virus testing and transfusion safety on the African continent. Transfusion 53(4): 816-826.

10. WHO (2017) Global hepatitis report. World Health Organization.

11. Owusu-Ofori A, Owusu-Ofori S (2015) Transfusion transmitted Malaria in Sub-Saharan Africa. ISBT Sciences Series 10(Suppl 1): 206-210.

12. Chitiyo ME (2011) Malaria and blood transfusion. Africa Sanguine 1256-1261.

13. Hume HA. Ddungu H, Angom R, Baluku H, Kajumbula H, et al, (2016) Platelet transfusion therapy in sub-Saharan Africa: bacterial contamination, recipient characteristics and acute transfusion reactions. Transfusion 56(8): 1951-1959.

14. Chaar MEl, Atwal S, Freimanis GL, Dinko B, Sutherland CJ, et al. (2013) Inactivation of Plasmodium falciparum in whole blood by riboflavin plus irradiation. Transfusion 53(12): 3174-3183.

15. The United States President's Emergency Plan for AIDS Relief.

16. WHO (2017) World Health Organization Regional Office for the Eastern Mediterranean (EMRO). Strategic 
Public Health Open Access

framework for blood safety and availability 2016-2025. Cairo: EMRO.

17. Tapko JB, Tagny CT (2021) The AfSBT step-wise accreditation program: Improving the quality in the
African blood services. Transfusion Clin Biol 28(2): 146147.

18. (2021) Africa Society for Blood Transfusion. AfSBT 23(1). 